\title{
Esculentoside A Attenuates Allergic Airway Inflammation via Activation of the Nrf-2 Pathway
}

\author{
Xinxin $\mathrm{Ci}^{\mathrm{a}}$ Weiting Zhong ${ }^{\mathrm{a}}$ Hua Ren ${ }^{\mathrm{a}, \mathrm{c}}$ Zhongmei Wen ${ }^{\mathrm{a}}$ Dan $\mathrm{Li}^{\mathrm{a}, \mathrm{b}}$ \\ Liping Peng ${ }^{a, b}$ \\ anstitute of Translational Medicine, and Departments of ${ }^{\mathrm{b}}$ Respiratory Medicine and ${ }^{\mathrm{c} O p h t h a l m o l o g y,}$ \\ The First Hospital, Jilin University, Changchun, China
}

\section{Key Words}

Esculentoside A · Asthma $\cdot$ Heme oxygenase $1 \cdot$ Nuclear

factor erythroid 2-related factor 2

\begin{abstract}
Background: The role of airway inflammation and inflammation-induced oxidative stress in the pathogenesis and progression of chronic inflammatory airway diseases has received increasing attention in recent years. We investigated the potential anti-inflammatory and antioxidative effects of esculentoside $A(E s A)$, a saponin isolated from the Chinese herb Phytolacca esculenta, in comparison to dexamethasone, a potent corticosteroid, in a murine model of allergic asthma. Methods: EsA was added to cultures of A549 cells at different concentrations or for different lengths of time, and nuclear factor erythroid 2-related factor 2 (Nrf-2) translocation and heme oxygenase 1 expression were monitored. Mice treated with or without EsA and Nrf-2 siRNA were sensitized and challenged with ovalbumin (OVA) and developed airway inflammation and oxidative lung damage. The Th2-type cytokine levels and inflammatory cells in bronchoalveolar lavage fluid (BALF) and the serum immunoglobulin production and adhesion molecule expression in the
\end{abstract}

lung tissues were measured. The activities of related antioxidases and glutathione were measured using assay kits. Results: EsA enhanced nuclear Nrf-2 translocation in both A549 cells and the lungs of OVA-challenged mice. Airway inflammation induced by OVA was reduced. Additionally, EsA increased mRNA expression of antioxidant enzymes regulated by Nrf-2, leading to a reduction in Th2 cytokines and the expression of adhesion molecule mRNA in the BALF and lung tissues. Inhibition of Nrf-2 by siRNA abrogated the regulatory effects of EsA on inflammation and oxidant stress. Conclusions: This is the first study to illustrate that EsA acts as a novel Nrf-2 activator, which modulates the oxidative stress pathway to improve lung injury and ameliorate the development of airway inflammation. @ $2015 \mathrm{~s}$. Karger AG, Basel

\section{Introduction}

The incidence of allergic airway diseases like asthma is rising prodigiously in both developed and developing countries. The classic hallmark of allergic asthma is airway inflammation, which is dominated by eosinophil infiltration and the production of mucus, Th2 cytokines

\section{KARGER 125}

(c) 2015 S. Karger AG, Base

$1018-2438 / 15 / 1674-0280 \$ 39.50 / 0$

E-Mail karger@karger.com

www.karger.com/iaa
Correspondence to: Dr. Dan Li or Prof. Liping Peng

Institute of Translational Medicine

The First Hospital, Jilin University

Changchun 130001 (China)

E-Mail 390336899@qq.com or plping1234@163.com 
(IL-4, IL-5 and IL-13) and allergen-specific IgE [1]. Oxidative stress can also initiate and augment inflammation, thus highlighting the importance of oxidants in asthma pathogenesis $[2,3]$. Airway epithelial cells play a key role in asthma, and act as the first line of defense against allergens entering the respiratory system by triggering the release of inflammatory mediators such as cytokines [4] and expressing adhesion molecules on their surfaces. In addition, reactive oxygen species (ROS) are generated in airway epithelial cells in response to a variety of stimuli, leading to airway inflammation characterized by airway narrowing, epithelium thickening, significant mucus secretion and inflammatory cell infiltration $[5,6]$.

The transcription factor, nuclear factor erythroid 2-related factor 2 (Nrf-2), is involved in lung defense against oxidative injury. After translocation to the nucleus, Nrf-2 induces the transcription of numerous antioxidant and cellular defense genes, including $\mathrm{NADP}(\mathrm{H})$ quinineoxidoreductase 1 (NQO-1), heme oxygenase 1 (HO-1), superoxide dismutase (SOD), catalase (CAT) and glutathione peroxidase [7]. Nrf-2 can be activated by diverse stimuli, including via the induction of cellular rescue pathways against oxidative damage, abnormal inflammation, immune responses, apoptosis and carcinogenesis [8-10]; this highlights the essential protective role of Nrf2 in protecting the lungs from disease. Disruption of the Nrf-2 gene leads to severe allergen-driven airway inflammation and hyperresponsiveness in mice and increased expression of the Th2 cytokines, IL-4 and IL-13, in the bronchoalveolar lavage fluid (BALF) and splenocytes after allergen challenge [11]. Nrf-2 also protects the lungs against the development of pulmonary fibrosis by regulating cellular redox levels and the Th1/Th2 balance in the lung [12-14].

Esculentoside A (EsA), also identified as 3-O-[ $\beta$ D-glucopyranosyl- $(1,4)-\beta$-D-xylopyranosyl] phytolaccagenin, is a saponin isolated from the root of Phytolacca esculenta. EsA can inhibit the production of proinflammatory cytokines, such as TNF- $\alpha$, IL- $1 \beta$, IL-2, IL- 6 and prostaglandin E2, in several cell types [15]. Zhang et al. [16] demonstrated that EsA attenuated acute liver injury in mice by inhibiting inflammatory responses and oxidative stress. Furthermore, EsA can suppress inflammatory responses in LPS-induced acute lung injury by inhibiting $\mathrm{NF}-\kappa \mathrm{B}$ overactivity and mitogen-activated protein kinase (MAPK) signaling pathways [17]. Given its reported antioxidant and anti-inflammatory properties, we speculated that EsA could modulate the oxidative stress pathway to protect against lung injury and ameliorate the development of allergic airway inflammation.

Antiallergic Inflammatory Effect of EsA

\section{Materials and Methods}

\section{Reagents}

EsA (purity, >92.2\%) was purchased from the National Institute for Food and Drug Control. The IL-4, IL-5 and IL-13 ELISA kits were purchased from BioLegend (California, USA). The kit for determining SOD, CAT and glutathione (GSH) activities was provided by the Beyotime Institute of Biotechnology (Nanjing, China). Antibodies against Nrf-2, HO-1 and $\beta$-actin were purchased from Cell Signaling (Boston, Mass., USA) or Abcam (Cambridge, Mass., USA). Dimethyl sulfoxide, DCF-DA and ovalbumin (OVA, grade V) were purchased from Sigma Chemical Co. (St. Louis, Mo., USA). Peroxidaseconjugated Affinipure goat anti-mouse $\lg \mathrm{G}(\mathrm{H}+\mathrm{L})$ and peroxidaseconjugated Affinipure goat anti-rabbit IgG $(\mathrm{H}+\mathrm{L})$ were purchased from PTG (Chicago, Ill., USA). TRIzol reagent was purchased from Invitrogen (Carlsbad, Calif., USA). FastStart Universal SYBR green master mix was purchased from Roche (Basel, Switzerland). A PrimeScript RT-PCR kit was purchased from Takara (Dalian, China).

\section{Cell Culture and Treatment}

A549, a human lung adenocarcinoma epithelial cell line, was obtained from the China Cell Line Bank (Beijing, China). Cells were cultured in Dulbecco's modified Eagle medium supplemented with $3 \mathrm{~mm}$ glutamine, antibiotics $(100 \mathrm{U} / \mathrm{ml}$ each of penicillin and streptomycin) and $10 \%$ heat-inactivated fetal bovine serum. The cells were maintained at $37^{\circ} \mathrm{C}$ in a humidified incubator containing $5 \% \mathrm{CO}_{2}$. In all experiments, cells were allowed to acclimate for $24 \mathrm{~h}$ before any treatments.

Western Blot Analysis of A549 Cells

A549 cells $\left(1 \times 10^{6} /\right.$ well $)$ were cultured in 6-well plates for $24 \mathrm{~h}$, and then treated for 3, 6 and $12 \mathrm{~h}$ with various concentrations of EsA. After each experiment, cells were washed twice with cold PBS and then scraped from the plates with $500 \mu \mathrm{l}$ of PBS. Cell homogenates were centrifuged at $3,000 \mathrm{~g}$ for $5 \mathrm{~min}$. Nuclear and cytoplasmic fractions of cells were prepared as previously described [18]. Whole-cell lysates were lysed in $1 \%$ non-diet P-40 lysis buffer $(1 \%$ $\mathrm{NP}-40,150 \mathrm{mM} \mathrm{NaCl}, 50 \mathrm{mM}$ Tris, pH 7.4) with freshly added protease and phosphatase inhibitors. After the lysates were incubated on ice for $30 \mathrm{~min}$, they were centrifuged $\left(12,000 \mathrm{~g}\right.$ at $\left.4^{\circ} \mathrm{C}\right)$ for $5 \mathrm{~min}$ to obtain the cytosolic fraction. Protein concentrations were determined using the Bradford assay before storage at $-80^{\circ} \mathrm{C}$. Cell lysates were subjected to immunoblotting analysis using the antibodies of anti-Nrf- 2 , anti-HO- 1 and anti- $\beta$-actin. The membranes were further probed with HRP-conjugated secondary antibodies and detected by ECL Western blot substrate. Band intensities were quantified using Image J gel analysis software. The fold increase in the level of protein expression was calculated by comparing it with that of normal controls. The experiments were repeated 3 times for each experimental condition.

\section{Animals}

Female BALB/c mice, weighing approximately 18-20 g, were purchased from Liaoning Changsheng Technology Industrial Co., Ltd (Certificate SCXK2010-0001; Liaoning, China). The mice were housed in microisolator cages and received food and water ad libitum. The laboratory temperature was $24 \pm 1^{\circ} \mathrm{C}$, and the relative humidity was $40-80 \%$. All animal studies were conducted according to the experimental practices and standards approved by the Animal Welfare and Research Ethics Committee at Jilin University. 
Table 1. Primer sets for RT-PCR analysis

\begin{tabular}{lll}
\hline Targets & Forward & Reverse \\
\hline HO-1 & CAGCCCCACCAAGTTCAAAC & AGGCG GTCTTAGCCTCTTCTG \\
NQO1 & AGGAAGAGCTAATAAATCTCTTCTTTGCTG & TCATATTGCAGATGTACGGTGTGGATTTAT \\
GST & AAGCCAGGA CTCTCACTA & AAGGCAGTCTTGGCTTCT \\
ICAM-1 & CTGGCTGTCACAGAACAGGA & AAAG-TAGGTGGGGAGGTGCT \\
VCAM-1 & CCCAAGGATCCAGA-GATTCA & TAAGGTGAGGGTGGCATTTC \\
Eotaxin & GAGCCTAAGAACTGCTTGATTCCT & GGGCGACTGGTGCTGATATT \\
$\beta$-Actin & CTAAGGCCAACCGTGAAAAG & AGCCTG-GATGGCTACGTACAT \\
\hline
\end{tabular}

Sensitization and Challenge with OVA and EsA

Mice were divided into the following study groups $(\mathrm{n}=15)$ : a nonsensitized control group, an OVA + EsA group, an OVA + dexamethasone (Dex) group and an OVA + EsA + siRNA group. All experiments were independently repeated 3 times. The nonsensitized control group was sensitized and challenged by intraperitoneal injection and inhalation of PBS. The mice in the other groups were each injected with $20 \mu \mathrm{g}$ of OVA adsorbed to $100 \mu \mathrm{g} /$ $\mathrm{ml}$ of Imject alum on days 0, 7 and 14 (general sensitization). On days 14 and 24-27, the mice were anesthetized and challenged with intranasal inhalation of $50 \mu \mathrm{g}$ OVA in $50 \mu \mathrm{l}$ PBS once daily. The mice in the OVA + EsA group were administered EsA intraperitoneally ( $15 \mathrm{mg} / \mathrm{kg} /$ day) $1 \mathrm{~h}$ prior to OVA administration on days 24-27, and the mice in the OVA + Dex group were administered Dex intraperitoneally ( $2 \mathrm{mg} / \mathrm{kg} /$ day $)$ on the same schedule. The mice in the OVA + EsA + siRNA group were intranasally administered Nrf-2-specific siRNA (si-Nrf-2; $5 \mathrm{nmol}$ in $200 \mu \mathrm{l}$ of PBS; Santa Cruz Biotechnology) $1 \mathrm{~h}$ before each EsA administration. On day 28, 5 mice were sacrificed for BALF collection, 5 for histopathological evaluation and enzymatic assays and 5 for RT-PCR and Western blot analysis.

\section{Collection of Blood and BAL}

At selected time points after the last inhalational exposure, the mice were anesthetized and bled via the brachial plexus for the collection of blood samples used to estimate the levels of total IgE and OVA-specific IgE. BAL was performed twice by intratracheal instillation of $500 \mathrm{ml}$ of PBS. The BALF was centrifuged and the supernatants used for cytokine and chemokine measurements. Cell pellets were resuspended in $1 \mathrm{ml}$ of PBS and used for total and differential cell counts as described [19].

\section{Lung Histology}

Histopathological evaluations were performed on mice. Left lungs were removed by dissection and fixed in $4 \%$ paraformaldehyde. Lung tissues were sectioned, embedded in paraffin and cut into 3-mm sections. Tissue sections were then stained with hematoxylin and eosin (HE) for general morphology [20] and with Alcian blue-periodic acid Schiff for the identification of goblet cells in the epithelium [21].

\section{Measurements of SOD, CAT and GSH Activities in Lung}

Tissues

The activities of SOD, CAT and GSH in the lung tissues were determined using enzymatic assay kits (Beyotime Institute of Biotechnology, Nanjing, China) according to the manufacturer's in- structions. Briefly, the lung tissues were snap-frozen in liquid nitrogen, lyophilized and homogenized. Supernatants were probed for enzymatic activity.

\section{Measurement of the Levels of Cytokines in BALF and IgE in} Serum

The levels of IL-4, IL-5 and IL-13 were quantified in BALF using sandwich ELISA kits (BioLegend, Calif., USA) according to the manufacturer's protocols. The levels of total IgE and OVA-specific IgE in the serum were also measured by ELISA. Microtiter plates were coated with or without $1 \%$ OVA in coating buffer $(0.05 \mathrm{M}$ sodium carbonate-bicarbonate, $\mathrm{pH} 9.6$ ) overnight at $4{ }^{\circ} \mathrm{C}$ and then incubated with samples of serum for $2 \mathrm{~h}$. The plates were then washed 4 times, and incubated with biotin anti-mouse IgE, followed by extravidin-peroxidase at room temperature for $30 \mathrm{~min}$, and with $3,3^{\prime}, 5,5^{\prime}$-tetramethylbenzidine substrate for $30 \mathrm{~min}$. The enzymatic reaction was stopped with $2 \mathrm{M} \mathrm{H}_{2} \mathrm{SO}_{4}$, and absorbance was read at $450 \mathrm{~nm}$. Concentrations were determined in duplicate for each sample.

\section{Measurement of ROS Production in the BALF}

The induction of oxidative stress was monitored using DCFDA (Molecular Probes), which is converted into highly fluorescent DCF by cellular peroxides, including hydrogen peroxide. Briefly, cells from BALF were washed with PBS, and the total cells $\left(5 \times 10^{4}\right)$ were counted. The BALF cells were treated with $10 \mu \mathrm{M}$ DCF-DA for $10 \mathrm{~min}$ at $37^{\circ} \mathrm{C}$. Intracellular ROS activity was measured by quantifying fluorescence at a $488-\mathrm{nm}$ excitation wavelength and a $525-\mathrm{nm}$ emission wavelength using a fluorescence plate reader.

\section{RNA Preparation and Quantitative RT-PCR}

Total RNA was isolated from the lungs (5 mice in each group) using the Trizol reagent (Invitrogen) according to the manufacturer's instructions. After the concentration of RNA was determined by spectrophotometer, $1 \mu \mathrm{g}$ of RNA was converted to cDNA by Prime-Script RT-PCR kit. Real-time PCR analysis was performed using the Applied Biosystems 7300 real-time PCR system and software (Applied Biosystems, Carlsbad, Calif., USA). Realtime PCR was conducted in 0.2-ml PCR tubes with forward and reverse primers and the SYBR green working solution, using a custom PCR master mix with the following conditions: at $95^{\circ} \mathrm{C}$ for $10 \mathrm{~min}$, followed by $40 \mathrm{cycles}$ at $95^{\circ} \mathrm{C}$ for $10 \mathrm{~s}$ and at $60^{\circ} \mathrm{C}$ for $30 \mathrm{~s}$. Primers are shown in table 1 . The relative gene expression was analyzed by normalizing with $\beta$-actin mRNA expression. A melting curve analysis was performed to control for the specificity of the amplification products. 
Fig. 1. EsA upregulated Nrf-2 and HO-1 expression. a, b A549 cells were treated with $20 \mathrm{mg} / \mathrm{l} \mathrm{EsA}$ for 3, 6 and 12 h. c, d A549 cells were treated with different concentrations of EsA for $6 \mathrm{~h}$. Nuclear and cytosolic fractions were separated and prepared for Western blot assays. Either GAPDH or Lamin B was used as a loading control; the protein level in each group was normalized against the loading control. The results are expressed as the mean \pm SEM $(n=3)$. ** $\mathrm{p}<0.01$ versus the control group.

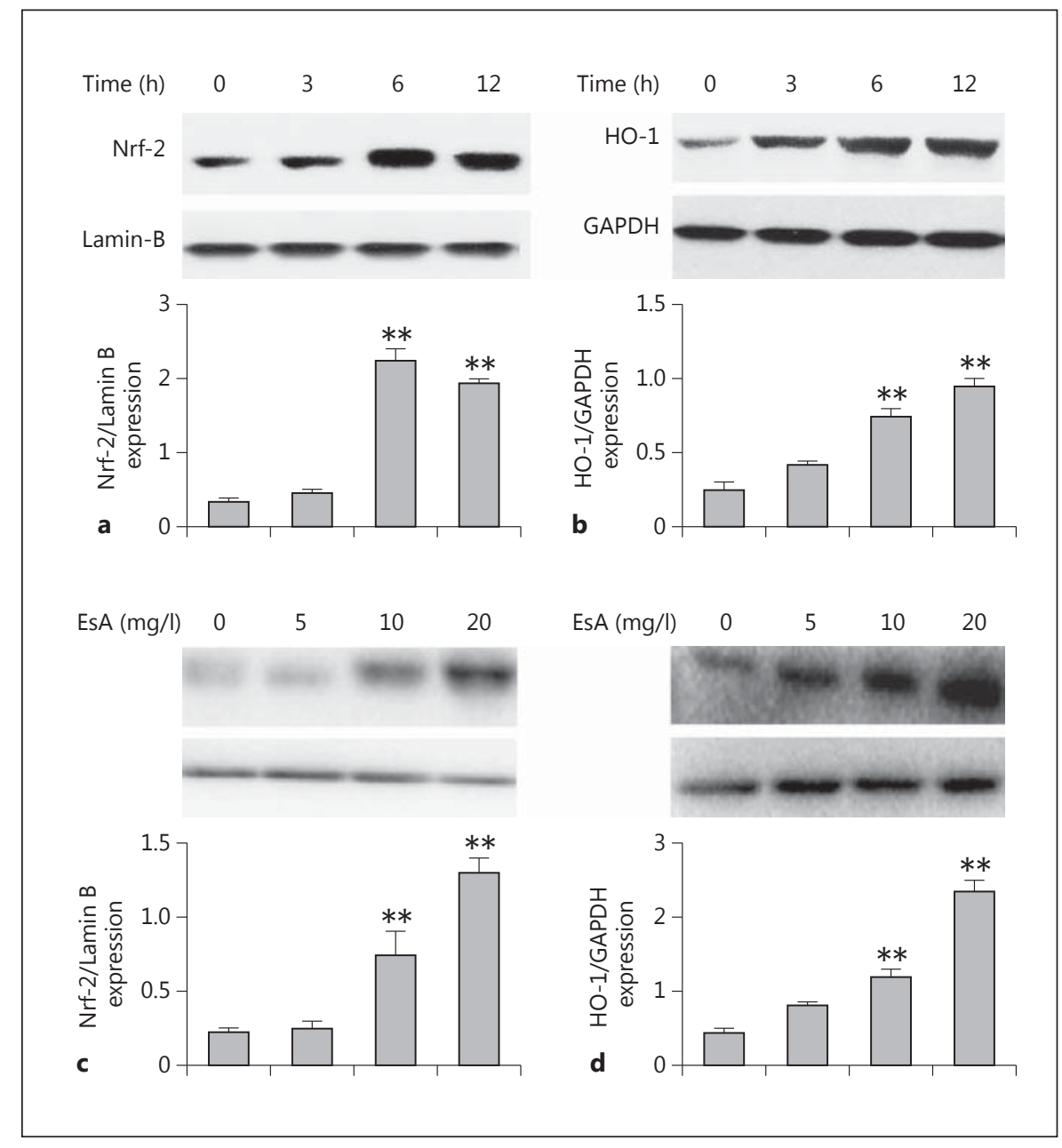

\section{Western Blot Analysis of Lung Tissue}

Lung tissues were added to lysis buffer and homogenized. Total protein extracts or nuclear extracts $(40 \mu \mathrm{g}$ per lane) were separated by $10 \%$ SDS-PAGE. Immunoblots were probed with antibodies against Nrf-2 and HO-1. $\beta$-Actin and Lamin B were used as internal controls for total protein extracts and nuclear extracts, respectively. Band intensity was quantitated using Image J software.

\section{Statistical Analysis}

Two-tailed unpaired Student's t tests were performed to determine $p$ values. All the graphs represent mean \pm SEM of 3 independent experiments, and asterisks represent $\mathrm{p}$ values, i.e. ${ }^{*} \mathrm{p}<0.05$ and $* * \mathrm{p}<0.01$.

\section{Results}

\section{EsA Facilitated Nrf-2 Translocation and Induced}

HO-1 Expression in A549 Cells

We first evaluated the antioxidative potential of EsA by measuring the localization of Nrf-2 protein and the expression of the antioxidant enzyme HO-1 in human lung epithelial cells. After treatment with EsA $(20 \mathrm{mg} / \mathrm{l})$ for 3, 6 and $12 \mathrm{~h}$, the translocation of Nrf-2 markedly increased, being most prominent at $6 \mathrm{~h}$ after treatment (fig. 1a). Furthermore, the expression of HO-1, a downstream molecule of $\mathrm{Nrf}-2$, also increased following EsA treatment, and was most prominent at $12 \mathrm{~h}$ after treatment (fig. 1b). We then investigated Nrf-2 translocation and $\mathrm{HO}-1$ expression after treatments with various concentrations of EsA for $6 \mathrm{~h}$, and both were enhanced in a dose-dependent manner (fig. 1c, d). These results demonstrated that EsA upregulates Nrf-2 and HO-1 in epithelial cells.

\section{EsA Decreases the Expression of Adhesion Molecules and Eotaxin in the Lung}

Next, we sought to further elucidate the effects of EsA on adhesion molecule production and eotaxin in vivo. In figure $2 \mathrm{a}$, it can be observed that OVA-challenged mice 


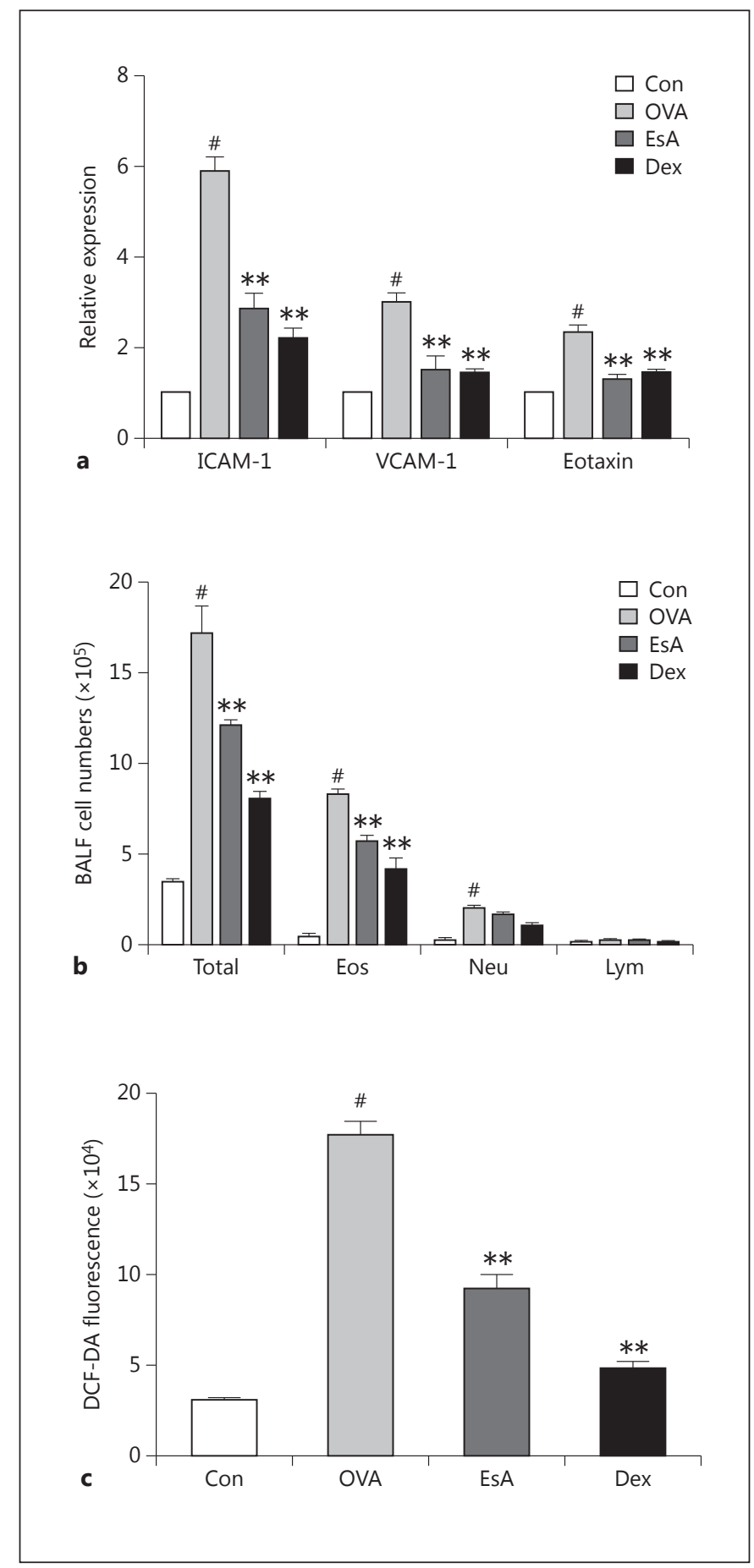

Fig. 2. EsA attenuated inflammatory mediator expression, immune cell infiltration and ROS production in mice. a Real-time PCR analysis of adhesion molecule and eotaxin expression in mouse lung tissue. $\beta$-Actin was used as a control. $\mathbf{b}$ Effects of EsA on cell numbers and differential cellular components in BALF. c Effects of EsA on ROS production in BALF. The results are expressed as the mean \pm SEM $(n=15) .{ }^{*} \mathrm{p}<0.05$ versus the control group; ${ }^{* *} \mathrm{p}<0.01$ versus the OVA-challenged group. Con $=$ Control; Eos = eosinophil; Lym = lymphocyte; $\mathrm{Neu}=$ neutrophil. had substantially higher levels of adhesion molecules and eotaxin in the lung than PBS-challenged mice. EsA reversed the elevation of VCAM-1, ICAM-1 and eotaxin levels induced by OVA.

\section{EsA Suppressed OVA-Induced Inflammatory Cell \\ Recruitment and ROS Production}

Inflammatory cell levels (i.e. total cells, eosinophils, neutrophils, macrophages and lymphocytes) were significantly elevated in BALF from OVA-challenged mice compared to that from control mice. The EsA-treated mice $(15 \mathrm{mg} / \mathrm{kg})$ had decreased total cell and eosinophils numbers (fig. 2b), compared with the OVA-challenged mice, but only slightly decreased infiltrating neutrophil numbers. Moreover, BALF from the OVA-challenged mice exhibited significantly increased ROS production compared with that from the negative control mice. BALF from the EsA-treated mice showed significantly decreased ROS production compared with that from the OVA-challenged mice (fig. 2c).

\section{EsA Alleviated Airway Inflammation, Goblet Cell \\ Hyperplasia and Mucus Production}

To assess the anti-inflammatory effect of EsA, histopathological studies were performed. Inflammatory cell infiltration into peribronchial and perivascular areas was observed in the OVA-challenged mice (HE), and was markedly reduced following EsA treatment $(15 \mathrm{mg} / \mathrm{kg}$; fig. 3a, c). To evaluate goblet cell hyperplasia, we stained lung sections with AB-PAS stain. Overproduction of mucus, goblet cell hyperplasia and epithelial cell disruption were observed in the bronchial airways of the OVA-treated mice but not in those of PBS-treated mice (fig. 3b, d). EsA $(15 \mathrm{mg} / \mathrm{kg})$ treatment significantly reduced mucus secretion, goblet cell hyperplasia and epithelial cell disruption.

\section{EsA Regulates OVA-Induced Th2 Cytokine Levels in} $B A L F$ and Serum Immunoglobulin Production in vivo

As shown in figure $4 \mathrm{a}$, the inhalation of OVA by sensitized mice caused a notable increase in the BALF IL-4, IL-5 and IL-13 concentrations compared with PBS-treated control mice. EsA (15 mg/kg) treatment significantly decreased the concentrations of IL-4, IL-5 and IL-13 $\left({ }^{*} \mathrm{p}<0.05,{ }^{* *} \mathrm{p}<0.01\right)$. To determine whether EsA could modify ongoing OVA-specific Th2 responses in vivo, total IgE and OVA-specific serum IgE levels were determined using ELISA. EsA ( $15 \mathrm{mg} / \mathrm{kg})$ significantly suppressed OVA-specific IgE, which was induced by OVA (fig. 4b). 
Fig. 3. EsA suppressed lung tissue eosinophilia and mucus production. Histologic examination. a Lung tissue eosinophilia. HE. b Mucus secretion. AB-PAS. Quantitative analyses of inflammatory cell infiltration (c) and mucus production (d) in lung sections were performed as previously described. At least 5 different fields for each lung section were scored for inflammatory cells and goblet cells. Mean scores were obtained from 15 mice. Con $=$ Control. ${ }^{* *} \mathrm{p}<$ 0.01 versus the OVA-challenged group.
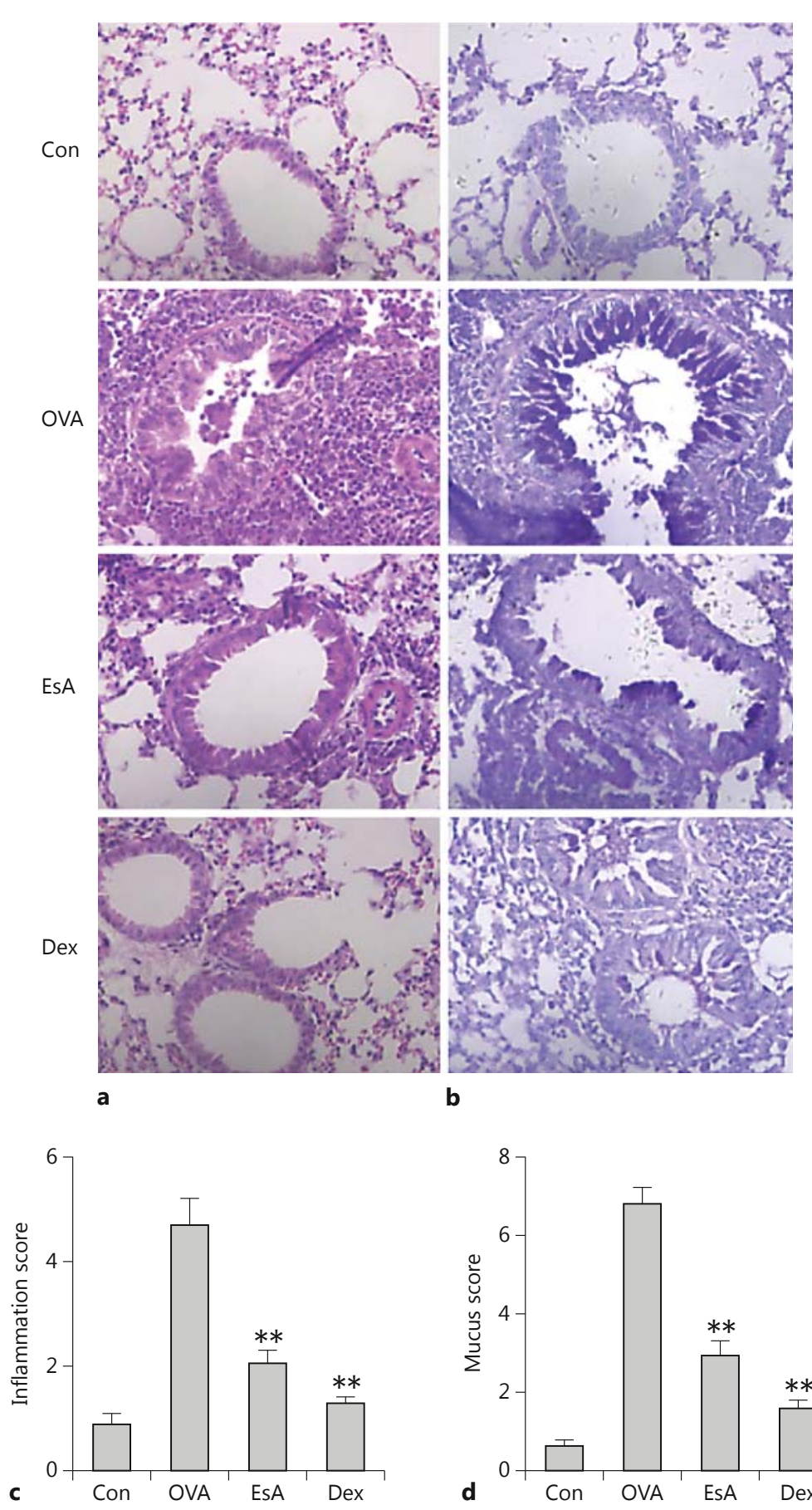

\section{EsA Enhances Antioxidant Capacity}

As shown in figure 5, the mice in both the OVA-sensitized and OVA-challenged groups showed significantly reduced levels of GSH, SOD and CAT activity in their
BALF; this was reversed by EsA treatment. Thus, EsA treatment reduces tissue oxidant stress and enhances endogenous antioxidant capacity. 

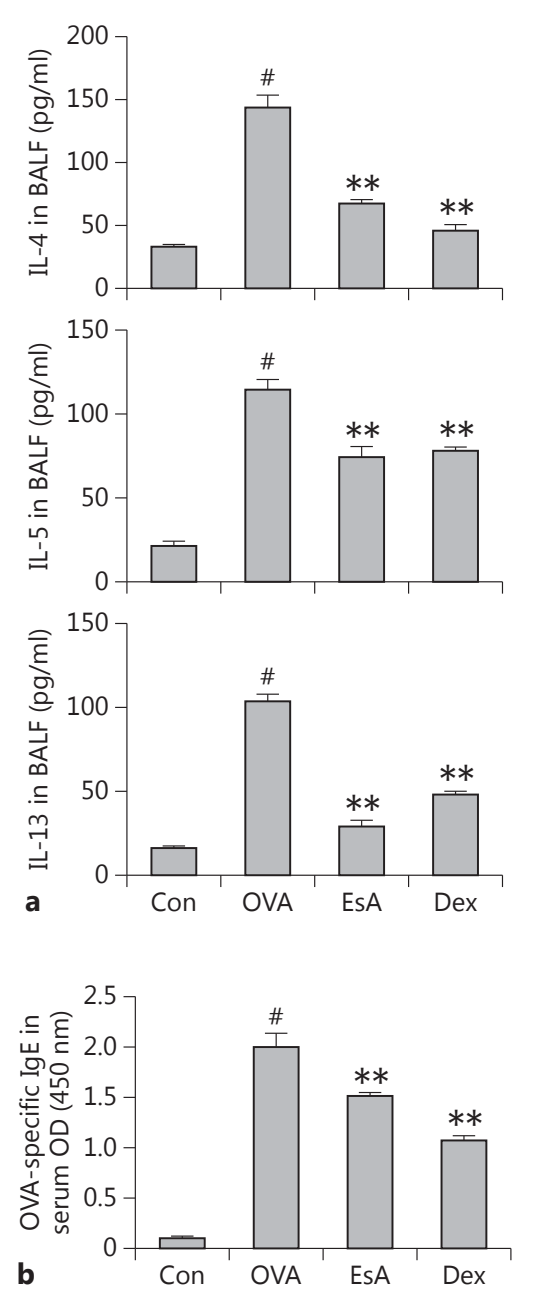

Fig. 4. EsA alleviated cytokine levels in BALF and serum immunoglobulin production in vivo. BALF and blood were collected and centrifuged $24 \mathrm{~h}$ after the last OVA challenge, and the supernatants and serum were measured by ELISA. Serum IgE (mean \pm SEM; $n=15$ ) levels are expressed as optical density (OD) values and are representative of at least 3 independent in vivo experiments. Con $=$ Control. ${ }^{\#} \mathrm{p}<0.05$ versus the control group; ${ }^{* *} \mathrm{p}<0.01$ versus the OVA-challenged group.

\section{EsA Exerts Antioxidant and Anti-Inflammatory}

\section{Effects via Nrf-2 Activation}

To demonstrate the involvement of Nrf-2 and downstream antioxidant enzyme activation in EsA stimulation, we next investigated $\mathrm{Nrf}-2$ expression following EsA treatment in the lung by Western blotting. EsA administration dramatically promoted Nrf- 2 translocation, ERK and p38 phosphorylation, but this effect was abolished in
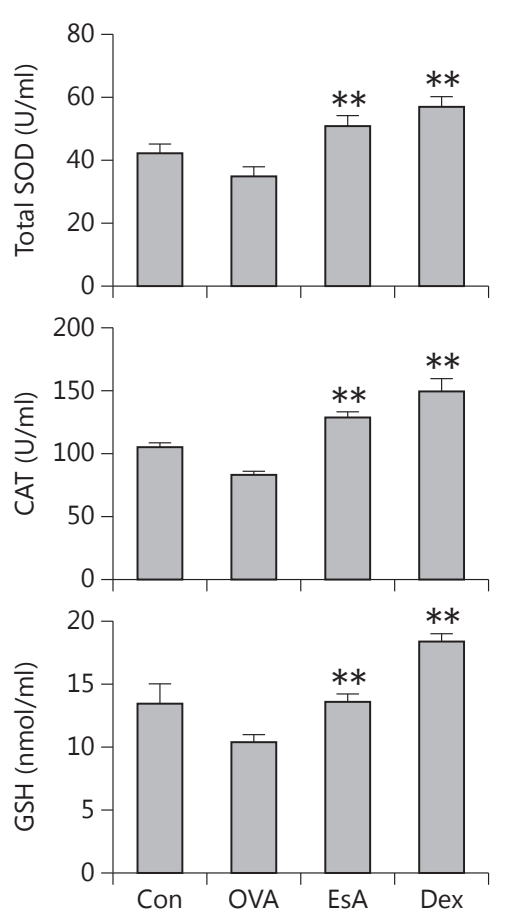

Fig. 5. EsA enhanced antioxidant capacity. Twenty-four hours after the final OVA challenge, BALF levels of total SOD, CAT and GSH activity were measured. Means \pm SEM of 15 mice/group. Con $=$ Control. ${ }^{* *} \mathrm{p}<0.01$ versus the OVA-challenged group.

Nrf-2 siRNA-treated mice (fig. 6a). The effects of EsA on the mRNA expression levels of Nrf-2 downstream genes in lung tissues of both the control and experimental groups of mice are displayed in figure $6 \mathrm{~b}$. The mRNA expression of HO-1, NQO-1 and GSH S-transferase (GST) increased in the lungs of the OVA-challenged mice following treatment with EsA and comparatively decreased following treatment with a combination of EsA and Nrf2 siRNA. The concentrations of the cytokines IL-4, IL-5 and IL-13 decreased in the lungs of the OVA-challenged mice following treatment with EsA and comparatively increased following treatment with a combination of EsA and Nrf-2 siRNA (fig. 6c, d).

\section{Discussion}

Airway inflammation is central to the pathogenesis of asthma and is caused by the presence of various factors, including ROS, proinflammatory mediators and growth 


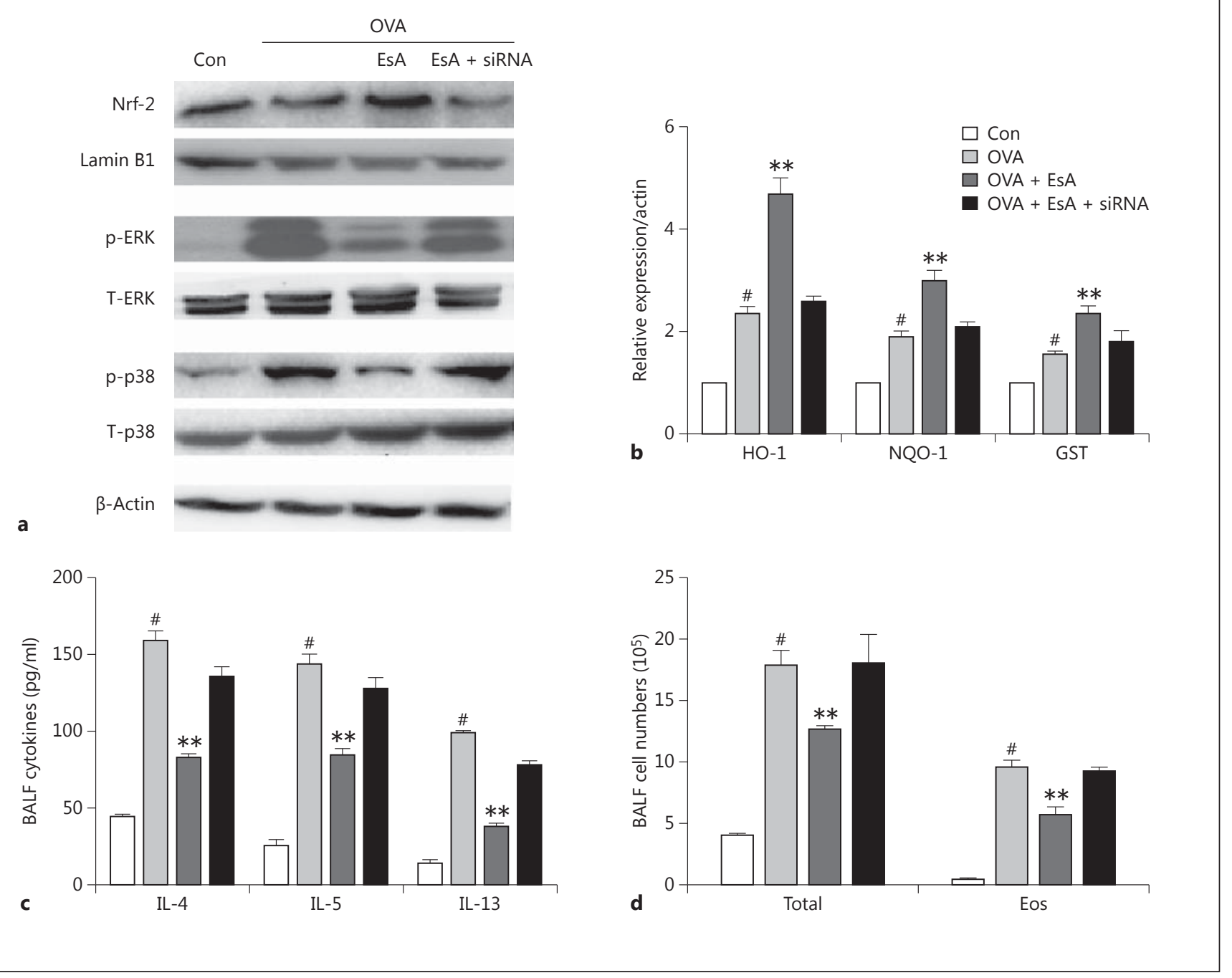

Fig. 6. Knockdown Nrf-2 abrogates the regulatory effects of EsA on the protein expression and downstream antioxidant enzymes in lung tissues as well as cytokine levels and inflammatory cell numbers in BALF. a EsA-mediated regulation of Nrf-2, p-ERK and p-p38 expression in protein extracts from lung tissues. $\mathbf{b}$ Real-time PCR analysis of antioxidant enzyme expression in mouse lung tis- sue. $\beta$-Actin was used as a control. c Knockdown Nrf-2 abrogates the inhibitory effects of EsA on cytokine levels in BALF. $\mathbf{d}$ Knockdown Nrf-2 abrogates the inhibitory effects of EsA on inflammatory cell numbers in BALF. The results are expressed as the mean $\pm \operatorname{SEM}(\mathrm{n}=15)$. Con $=$ Control. ${ }^{\#} \mathrm{p}<0.05$ versus the control group; ${ }^{* *} \mathrm{p}<0.01$ versus the OVA-challenged group. factors [22]. The role of inflammation-induced oxidative stress in the pathogenesis and progression of chronic inflammatory airway diseases such as asthma has received increasing attention in recent years. Epithelial cells play a central role in regulating airway inflammation and oxidative stress. The results from our in vitro study showed that EsA induced the expression of antioxidant proteins such as $\mathrm{HO}-1$ and enhanced Nrf-2 protein translocation from the cytoplasm to the nucleus in A549 cells, thereby dem- onstrating the antioxidative potential of EsA. Using an OVA-challenged murine asthmatic model, we further demonstrated that both EsA and Dex (used as a control) markedly attenuated asthmatic responses, including airway inflammation, eosinophil migration into the lung, mucus hypersecretion and reductions in Th2 cytokines and IgE, while enhancing antioxidant capacity by upregulating SOD, CAT and GSH. The results of the Dex control group raise the question of whether EsA exerts an 
Fig. 7. The proposed mechanism of EsAmediated amelioration of airway inflammation: Nrf-2 is activated in response to allergen challenge in the lungs of EsA-treated mice, resulting in the induction of several antioxidant genes that may protect against airway inflammation, immune cell infiltration and Th2 cytokine release.

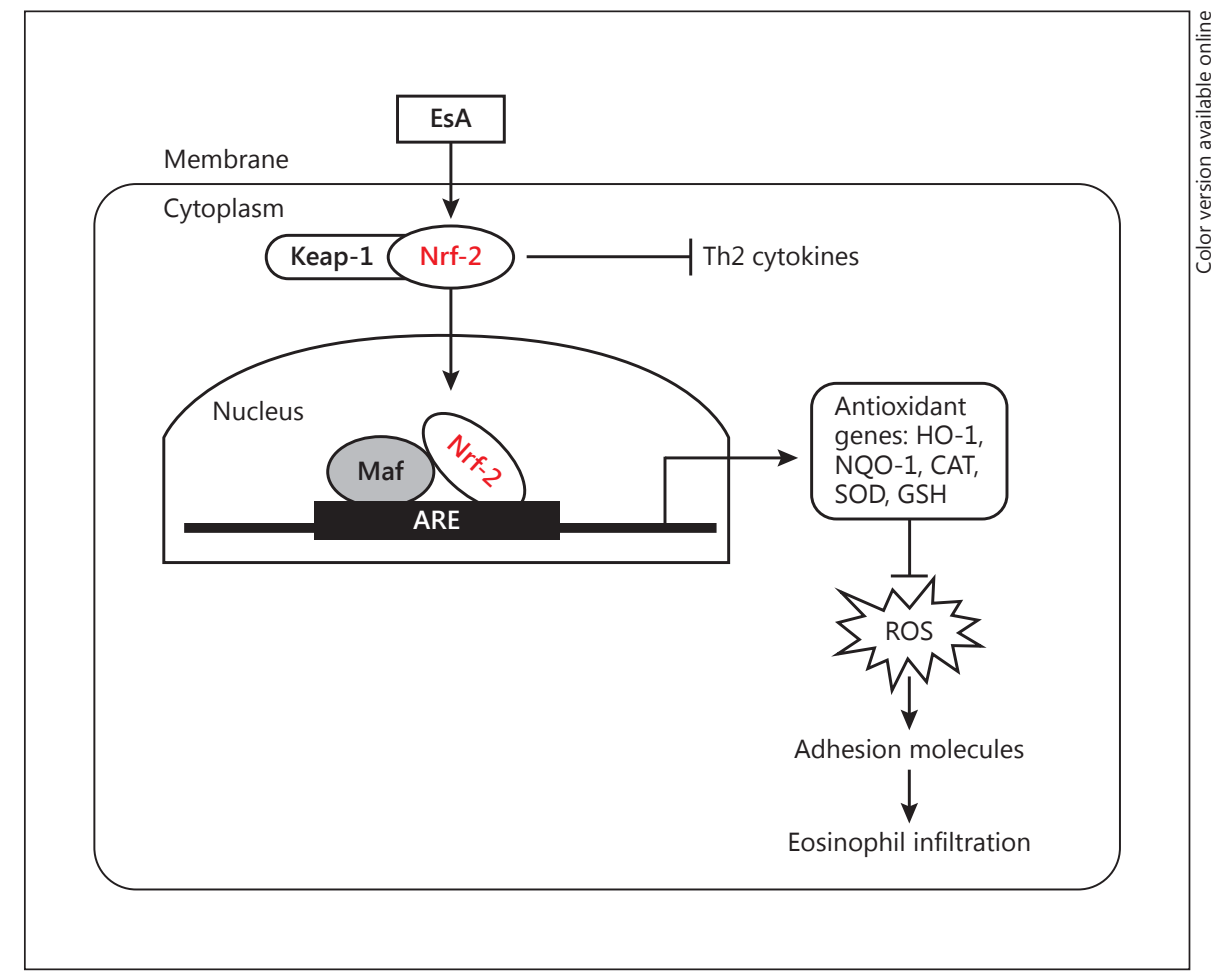

anti-inflammatory effect that is followed by decreased oxidative stress or vice versa. Using Nrf-2 siRNA, we further demonstrated that EsA could attenuate airway inflammation and enhance the antioxidant response in OVA-challenged asthmatic mice. The combined activation of the Nrf-2 pathway and suppression of oxidative stress may underlie the anti-inflammatory and tissueprotective effects of EsA.

Normally, low levels of oxidative stress are well tolerated due to the protection afforded by antioxidants that are present in the lungs, such as SOD, CAT and GSH [23]. High levels of oxidative stress eventually impair pulmonary antioxidant defenses. In patients with severe asthma, oxidative stress is a major contributing factor to resistance to steroid therapy [24]. There is an urgent need to discover new drug compounds that possess both anti-inflammatory and antioxidant properties for the treatment of chronic severe asthma.

Nrf-2, a redox-sensitive transcription factor, plays an important role in inducing the expression of antioxidant response element (ARE)-dependent genes, including phase II enzymes [25]. Previous studies have suggested that Nrf- 2 has indirect modulatory effects on various immune and inflammatory pathways. Bronchial epithelial cells have been shown to possess robust antioxidant de- fenses against environmental insults via the activation of Nrf-2 [26]. Disruption of the Nrf-2 gene also leads to increased expression of the Th2 cytokines, IL-4 and IL-13, in both the BALF and splenocytes after allergen challenge, indicating the important protective role of the Nrf-2 pathway in allergic and asthmatic responses in vivo [12]. Indeed, recent studies employing dendritic cells in a mouse model demonstrated that Nrf- 2 protects against allergic airway inflammation following exposure to different stimuli in vivo [27, 28]. Our current results showed that EsA enhanced Nrf-2 protein translocation from the cytoplasm to the nucleus and upregulated HO-1 expression both in A549 cells and lung tissue from OVA-challenged mice.

The OVA-challenged mice displayed pronounced mucus overproduction and inflammatory cell infiltration into lung tissues compared with the negative-control mice. Eosinophils have been identified as the major effector cell type underlying allergic airway inflammation [29], and excess mucus production and increased goblet cell differentiation are characteristic of this condition. Our data show that EsA treatment significantly reduced eosinophil numbers in the BALF as well as inflammatory cell infiltration in the peribronchial and perivascular areas, mucus overproduction and goblet cell hyperplasia. 
Both the production of allergen-specific IgE and the overexpression of Th2-mediated cytokines, including IL-4, IL-5 and IL-13, play an essential role in the pathogenesis of allergic airway inflammation. OVA exposure in the airway produces a Th2-dominant response by activating inflammatory cells and upregulating IL-4, IL-5 and IL-13 secretion (fig. 4). EsA treatment reduced serum levels of OVA-specific IgE and BALF levels of the Th2 cytokines IL-4, IL-5 and IL-13. Furthermore, levels of the adhesion molecules, ICAM-1 and VCAM-1, and the chemokine eotaxin, were substantially reduced.

Dietary or pharmacological antioxidants that enhance nonenzymatic defenses have been extensively investigated in lung disease. Growing evidence suggests that the health benefits of dietary compounds such as curcumin, quercetin and catechin are dependent on the activation of Nrf-2 signaling [30]. The restoration of antioxidant and detoxifying enzymes by Nrf-2 provides an attractive therapeutic approach to target airway inflammation. EsA, a saponin isolated from the root of $P$. esculenta, has the ability to attenuate acute liver injury in mice via the inhibition of inflammatory responses and oxidative stress. In our study, OVA-challenged mice exhibited reduced levels of antioxidants, including SOD, CAT and GSH. EsA treatment significantly increased the levels of antioxidants and detoxifying enzymes (HO-1, NQO- 1 and GST). More importantly, the inhibition of Nrf-2 by siRNA ab- rogated the regulatory effect of EsA on inflammation (downregulation of Th2 cytokines and inflammatory cell numbers), oxidant stress (upregulation of detoxifying enzymes) and the ERK and p38 pathways.

In conclusion, these results provide a clear link between airway inflammation and defective Nrf-2 signaling (fig. 7). Nrf-2 was activated in response to allergen challenge in the lungs of EsA-treated mice, resulting in the induction of several antioxidant genes that may protect against airway inflammation, immune cell infiltration and Th2 cytokine release. Conversely, defective Nrf- 2 signaling may abolish the protective effect of EsA on severe lung inflammation. Collectively, we have demonstrated that EsA may exert anti-inflammatory effects and thereby ameliorate the development of airway inflammation by modulating the oxidative stress pathway.

\section{Acknowledgements}

This work supported by a grant from the Natural Science Foundation of Jilin (No. 20150520050JH).

\section{Disclosure Statement}

There are no conflicts of interest.

\section{References}

1 Busse WW, Lemanske RF Jr: Asthma. N Engl J Med 2001;344:350-362.

2 Hoshino T, Okamoto M, Takei S, Sakazaki Y, Iwanaga T, Aizawa H: Redox-regulated mechanisms in asthma. Antioxid Redox Signal 2008;10:769-783.

3 Sugiura $\mathrm{H}$, Ichinose M: Oxidative and nitrative stress in bronchial asthma. Antioxid Redox Signal 2008;10:785-797.

4 Holgate ST: The airway epithelium is central to the pathogenesis of asthma. Allergol Int 2008;57:1-10.

5 Bloemen K, Verstraelen S, Van Den Heuvel R, Witters H, Nelissen I, Schoeters G: The allergic cascade: review of the most important molecules in the asthmatic lung. Immunol Lett 2007;113:6-18.

6 Hsu WH, Lee BH, Huang YC, Hsu YW, Pan TM: Ankaflavin, a novel Nrf-2 activator for attenuating allergic airway inflammation. Free Radic Biol Med 2012;53:1643-1651.

7 Nguyen T, Nioi P, Pickett CB: The Nrf2-antioxidant response element signaling pathway and its activation by oxidative stress. J Biol Chem 2009;284:13291-13295.
8 Walters DM, Cho HY, Kleeberger SR: Oxidative stress and antioxidants in the pathogenesis of pulmonary fibrosis: a potential role for Nrf2. Antioxid Redox Signal 2008;10:321-332.

$9 \mathrm{Wu} J, \mathrm{Xu} \mathrm{H}$, Wong PF, Xia S, Xu J, Dong J: Icaritin attenuates cigarette smoke-mediated oxidative stress in human lung epithelial cells via activation of PI3K-AKT and Nrf2 signaling. Food Chem Toxicol 2014;64:307-313.

10 Hamed EA, Zakhary MM, Maximous DW: Apoptosis, angiogenesis, inflammation, and oxidative stress: basic interactions in patients with early and metastatic breast cancer. J Cancer Res Clin Oncol 2012;138:999-1009.

11 Rangasamy T, Guo J, Mitzner WA, Roman J, Singh A, Fryer AD, Yamamoto M, Kensler TW, Tuder RM, Georas SN, Biswal S: Disruption of Nrf2 enhances susceptibility to severe airway inflammation and asthma in mice. J Exp Med 2005;202:47-59.

12 Cho HY, Kleeberger SR: Noblesse oblige: NRF2 functions in the airways. Am J Respir Cell Mol Biol 2014;50:844-847.
13 Kikuchi N, Ishii Y, Morishima Y, Yageta Y, Haraguchi N, Itoh K, Yamamoto M, Hizawa $\mathrm{N}$ : Nrf2 protects against pulmonary fibrosis by regulating the lung oxidant level and Th1/ Th2 balance. Respir Res 2010;11:31.

14 Cho HY, Reddy SP, Yamamoto M, Kleeberger SR: The transcription factor NRF2 protects against pulmonary fibrosis. FASEB J 2004; 18 : 1258-1260.

15 Xiao ZY, Zheng QY, Jiang YY, Zhou B, Yin M, Wang HB, Zhang JP: Effects of esculentoside A on production of interleukin-1,2, and prostaglandin E2. Acta Pharmacol Sin 2004;25: 817-821.

16 Zhang F, Wang X, Qiu X, Wang J, Fang H, Wang Z, Sun Y, Xia Z: The protective effect of esculentoside A on experimental acute liver injury in mice. PLoS One 2014;9:e113107.

17 Zhong WT, Jiang LX, Wei JY, Qiao AN, Wei MM, Soromou LW, Xie XX, Zhou X, Ci XX, Wang DC: Protective effect of esculentoside A on lipopolysaccharide-induced acute lung injury in mice. J Surg Res 2013;185:364-372. 
18 Ito K, Jazrawi E, Cosio B, Barnes PJ, Adcock IM: p65-activated histone acetyltransferase activity is repressed by glucocorticoids: mifepristone fails to recruit HDAC2 to the p65HAT complex. J Biol Chem 2001;276:3020830215.

19 Rogerio AP, Fontanari C, Borducchi E, Keller AC, Russo M, Soares EG, Albuquerque DA, Faccioli LH: Anti-inflammatory effects of $\mathrm{La}$ foensia pacari and ellagic acid in a murine model of asthma. Eur J Pharmacol 2008;580: 262-270.

20 McKay A, Leung BP, McInnes IB, Thomson NC, Liew FY: A novel anti-inflammatory role of simvastatin in a murine model of allergic asthma. J Immunol 2004;172:2903-2908.

21 Edwan JH, Perry G, Talmadge JE, Agrawal DK: Flt-3 ligand reverses late allergic response and airway hyper-responsiveness in a mouse model of allergic inflammation. J Immunol 2004;172:5016-5023.
22 Lee MY, Seo CS, Lee JA, Lee NH, Kim JH, Ha $\mathrm{H}$, Zheng MS, Son JK, Shin HK: Anti-asthmatic effects of Angelica dahurica against ovalbumin-induced airway inflammation via upregulation of heme oxygenase-1. Food Chem Toxicol 2011;49:829-837.

23 MacNee W: Oxidative stress and lung inflammation in airways disease. Eur J Pharmacol 2001;429:195-207.

24 Barnes PJ: Corticosteroid resistance in airway disease. Proc Am Thorac Soc 2004;1:264-268.

$25 \mathrm{Na} \mathrm{HK}$, Surh YJ: Oncogenic potential of Nrf2 and its principal target protein heme oxygenase-1. Free Radic Biol Med 2014;67:353-365.

26 Li N, Alam J, Venkatesan MI, Eiguren-Fernandez A, Schmitz D, Di Stefano E, Slaughter $\mathrm{N}$, Killeen E, Wang X, Huang A, Wang $M$, Miguel AH, Cho A, Sioutas C, Nel AE: Nrf2 is a key transcription factor that regulates antioxidant defense in macrophages and epithelial cells: protecting against the proinflammatory and oxidizing effects of diesel exhaust chemicals. J Immunol 2004;173:3467-3481.
27 Williams MA, Rangasamy T, Bauer SM, Killedar S, Karp M, Kensler TW, Yamamoto M, Breysse P, Biswal S, Georas SN: Disruption of the transcription factor Nrf2 promotes prooxidative dendritic cells that stimulate Th2like immunoresponsiveness upon activation by ambient particulate matter. J Immunol 2008;181:4545-4559.

28 Rangasamy T, Williams MA, Bauer S, Trush MA, Emo J, Georas SN, Biswal S: Nuclear erythroid 2 p45-related factor 2 inhibits the maturation of murine dendritic cells by ragweed extract. Am J Respir Cell Mol Biol 2010;43: 276-285.

29 Hogan SP, Rosenberg HF, Moqbel R, Phipps S, Foster PS, Lacy P, Kay AB, Rothenberg ME: Eosinophils: biological properties and role in health and disease. Clin Exp Allergy 2008;38: 709-750.

30 Kumar H, Kim IS, More SV, Kim BW, Choi DK: Natural product-derived pharmacological modulators of Nrf2/ARE pathway for chronic diseases. Nat Prod Rep 2014;31:109-139. 